\title{
Infantile hemangioma of the liver in an adult: A case report and review of the literature
}

\author{
AKIKO SUMI $^{1,2}$, JUN AKIBA $^{1}$, SHUJI NAGATA $^{2}$, TATSUYUKI TONAN $^{2}$, \\ YORIKO NOMURA $^{1,3}$, TOSHIRO OGATA ${ }^{3}$, MASAFUMI UCHIDA ${ }^{4}$, \\ OSAMU NAKASHIMA ${ }^{5}$, MASAYOSHI KAGE ${ }^{1}$ and HIROHISA YANO ${ }^{1}$
}

\author{
Departments of ${ }^{1}$ Pathology, ${ }^{2}$ Radiology and ${ }^{3}$ Surgery, Kurume University School of Medicine, Kurume, \\ Fukuoka 830-0011; ${ }^{4}$ Department of Radiology, Kurume University Medical Center, Kurume, Fukuoka 839-0863; \\ ${ }^{5}$ Department of Clinical Laboratory Medicine, Kurume University Hospital, Kurume, Fukuoka 830-0011, Japan
}

Received April 24, 2013; Accepted October 14, 2013

DOI: $10.3892 / \mathrm{mmr} .2013 .1749$

\begin{abstract}
Infantile hemangioma (IH), a representative vascular liver tumor, usually occurs in infancy or early childhood but rarely in adults. In this study, we describe a case of $\mathrm{IH}$ in a 47 -year-old female and we also review the literature. A plain computed tomography (CT) image revealed five hypoattenuating masses in the liver. A dynamic study revealed the masses appeared to be well-enhanced in the arterial phase, and were considered to be high-flow hemangiomas. The tumors appeared as hypointense tumors on the T1-weighted images and as hyperintensities on fat-suppression $\mathrm{T} 2$-weighted images. Following the administration of gadolinium ethoxybenzyl diethylenetriamine pentaacetic acid (Gd-EOB-DTPA), tumors appeared to be well-enhanced in the arterial phase. In the portal phase, tumors demonstrated isointensity compared with the surrounding liver parenchyma, and hypointensity in the equilibrium and hepatobiliary phases. The apparent diffusion coefficient (ADC) values ranged from 2.0 to $2.4 \times 10^{-3} \mathrm{~mm}^{2} / \mathrm{sec}$. Microscopically, the tumors were composed of numerous capillary-like small vessels lined with plump endothelial cells, arranged in a single layer without mitoses, and small bile ducts were trapped and scattered within the tumor. These findings were considered to be characteristic of $\mathrm{IH}$. To the best of our knowledge, this case is the third report on IH in adults.
\end{abstract}

\section{Introduction}

Hemangiomas are the most common type of benign hepatic tumor. In adults, the major histological type is a cavernous hemangioma. By contrast, infantile hemangioma (IH), also referred to as infantile hemangioendothelioma, is a vascular

Correspondence to: Dr Akiko Sumi, Department of Pathology, Kurume University School of Medicine, 67 Asahimachi, Kurume, Fukuoka 830-0011, Japan

E-mail: sumi_akiko@kurume-u.ac.jp

Key words: hemangioma, infantile, adult, EOB-MRI, pathology liver tumor arising in infancy or early childhood. The majority of cases are diagnosed by the age of six months. IH in adults is extremely rare, and only a few cases have been reported in the English literature thus far. The present review reports the case of a vascular tumor, morphologically resembling $\mathrm{IH}$, that presented in a 47-year-old female, and a review of the literature.

\section{Case report}

A 47-year-old female visited Koga hospital for a medical check. An abdominal ultrasound revealed five tumors in the right lobe of the liver. Laboratory studies, including liver function tests and tumor markers, were within the normal range. Serological markers for hepatitis $\mathrm{B}$ or $\mathrm{C}$ viral infections were undetectable.

Abdominal computed tomography (CT) and gadolinium ethoxybenzyl diethylenetriaminepentaacetic acid (Gd-EOB-DTPA) enhanced magnetic resonance imaging (MRI) findings, which are shown in Figs. 1 and 2, respectively. A plain CT image revealed five hypoattenuating lesions in the right lobe of the liver. The arterial phase of the dynamic CT revealed marked enhancement of the tumors. The largest tumor had no enhanced area within the tumor considered to be necrosis or cystic/mucinous degeneration. The tumors showed hyperattenuation compared with the surrounding liver parenchyma in the delayed phase (Fig. 1). Tumors appeared as hypointense masses on the T1-weighted images and as hyperintensities on the fat-suppression T2-weighted images. Following the administration of Gd-EOB-DTPA, the tumors appeared to be well-enhanced in the arterial phase $(30 \mathrm{sec})$. In the portal phase $(80 \mathrm{sec})$, the tumors demonstrated isointensity compared with the surrounding liver parenchyma, and hypointensity in the equilibrium (4 $\mathrm{min})$ and hepatobiliary $(20 \mathrm{~min})$ phases. In the CT, the largest tumor had no enhanced area within the tumor. The apparent diffusion coefficient (ADC) values ranged from 2.0 to $2.4 \times 10^{-3} \mathrm{~mm}^{2} / \mathrm{sec}$ (Fig. 2).

Clinically, these tumors were considered to be hemangiomas. As the tumors were enlarged during follow-up, a liver biopsy was performed, and the tumors were diagnosed histologically as hemangiomas. 
The patient was followed up without therapy for $\sim 4$ years, but the tumors increased in size. As a result, the patient underwent a lobectomy of the right liver.

Grossly, the tumors were well defined and brown to tan, and the largest tumor measured 66x57 $\mathrm{mm}$ in diameter with a yellowish area considered to be myxoid degeneration (Fig. 3). Microscopically, the tumors consisted of numerous capillarylike vessels lined with endothelial cells without atypia or mitoses. A myxomatous component was noted in the center of the largest tumor. Immunohistochemically, the endothelial cells were positive for CD34 and vimentin, and negative for Glut-1, HMB45 and CAM5.2. The small bile ducts trapped within the tumor were positive for CD56 (Fig. 4).

Clinically, no apparent recurrence was noted in the subsequent 12 months.

\section{Discussion}

IH is one of the most common benign vascular liver tumors in infants and children. Most IHs are diagnosed before six months of age, and enlarge in the first 8-18 months $(1,2)$. Following this period, the occurrence of $\mathrm{IH}$ is exceedingly rare. There are only two adult case reports of $\mathrm{IH}$, occurring in a 54-year-old female and an 18-year-old adolescent $(1,3)$. The majority of patients present with abdominal distention and certain patients present with congestive heart failure or consumption coagulopathy $(1,4)$. Selby et al reported that the most prognostically important symptoms are congestive heart failure and jaundice (1). Up to two-thirds of symptomatic patients die as a result of the tumor. If patients harboring IH do not have any complications, the majority of tumors spontaneously regress with age $(5,6)$. In the present case, the patient had no symptoms or complications although the tumors gradually increased in size.

MRI findings of IH have been previously reported. Although MRI findings reveal various intensities due to hemorrhaging, infarction or degeneration, the tumor shows heterogeneous hypointensity relative to the surrounding liver parenchyma on T1-weighted images and heterogeneous hyperintensity on T2-weighted images. These findings are similar to those of cavernous hemangioma (7,8-11). In the present case, the tumors demonstrated similar signal intensities to those identified in the previous reports.

To the best of our knowledge, there have been no previous reports that present Gd-EOB-DTPA-enhanced MRI image findings of IH. However, there is a report on the enhancement pattern in a dynamic study with Gd-EOB-DTPA of typical and high-flow hemangiomas (12). In typical cases, tumors demonstrate peripheral nodular enhancement and a progressive fill-in pattern in the arterial phase, although high-flow type hemangiomas exhibit uniform enhancement in the arterial phase. Tamada et al reported that $59 \%$ of hemangiomas were isointense during the portal phase and $69 \%$ were isointense to slightly hypointense during the equilibrium phase. In the hepatobiliary phase $(20 \mathrm{~min})$, all the tumors demonstrated hypointensity relative to the surrounding liver parenchyma and there were no differences in these enhancement patterns between typical hemangiomas and high-flow hemangiomas (12). However, particularly with large lesions, tumors contain unenhanced areas due to infarctions or
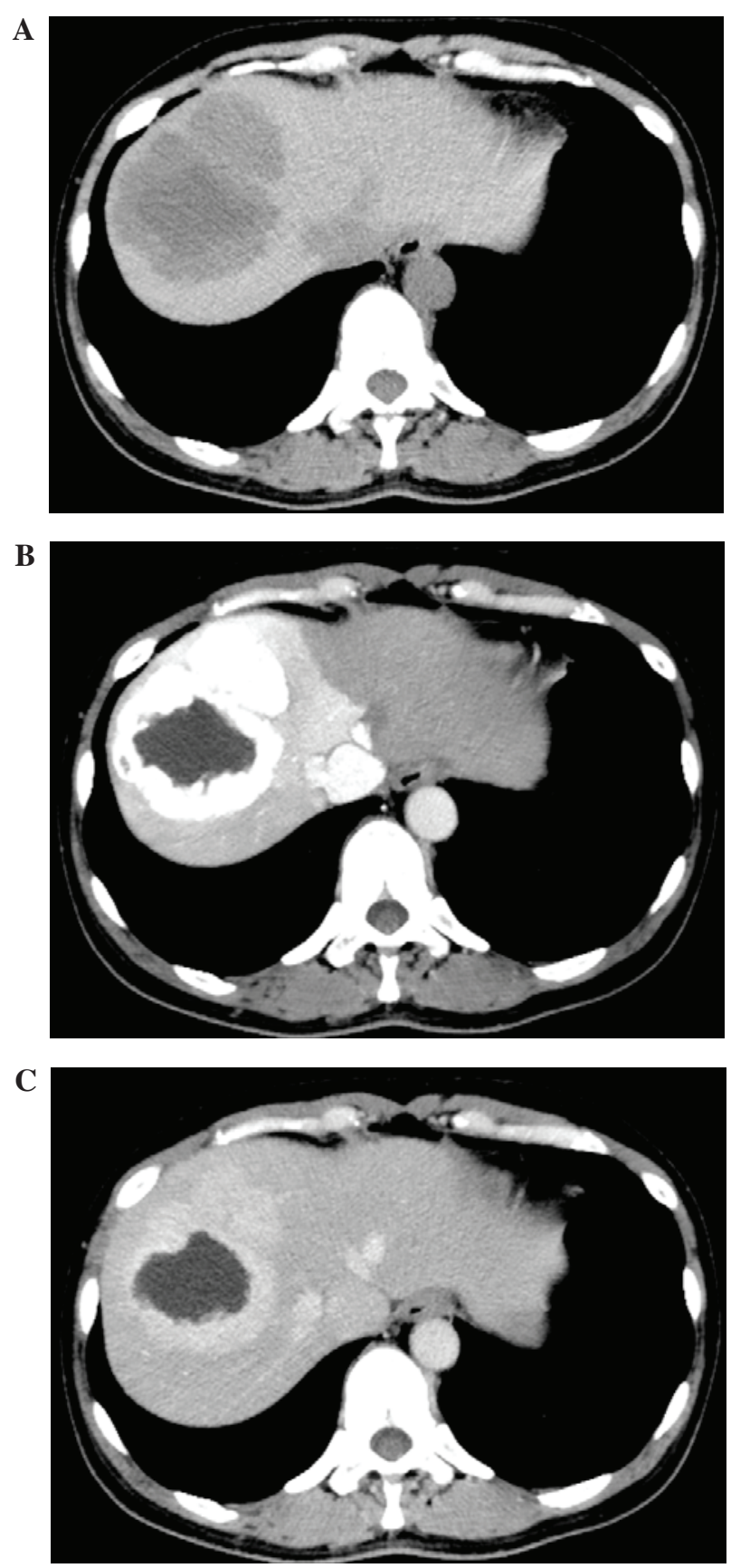

Figure 1. Computed tomography (CT) image of infantile hemangioma in hepatic segment VIII. (A) Plain CT image revealing hypoattenuating lesions in the right lobe of the liver. (B) Tumor shows homogenous enhancement in the arterial phase. (C) In the delayed phase, the tumor shows hyperattenuation relative to the surrounding liver parenchyma and there is no enhanced area inside the tumor.

hemorrhaging $(8,9)$. The present case was considered to be a high-flow-type hemangioma and the largest tumor had a non-enhancement area that was identified microscopically as a myxoid change.

Although no previous reports have demonstrated the DWI or ADC value of IH, certain reports have described that of hemangioma in adult cases. Vossen et al reported that there was a statistically significant difference in the ADC values between hemangiomas and other hypervascular liver lesions (focal nodular hyperplasia, hepatocellular carcinoma and hypervascular liver metastases) (13). Taouli et al also reported 
A

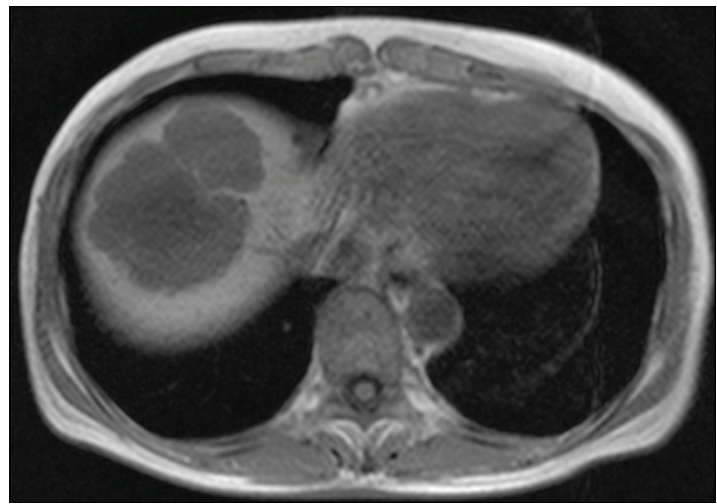

C

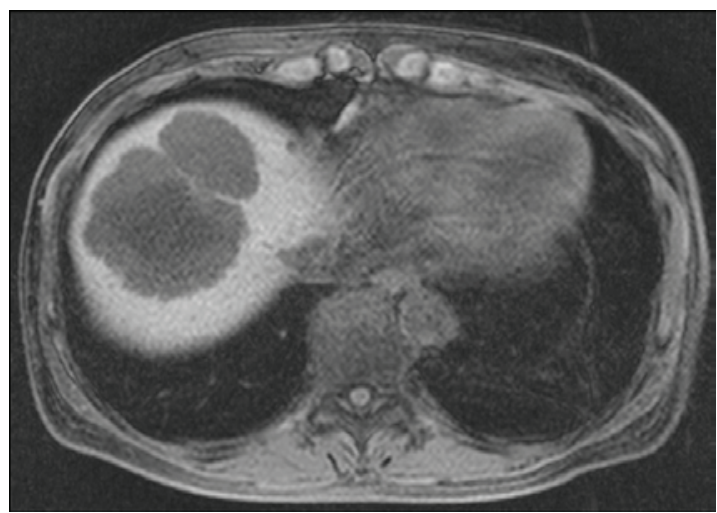

E

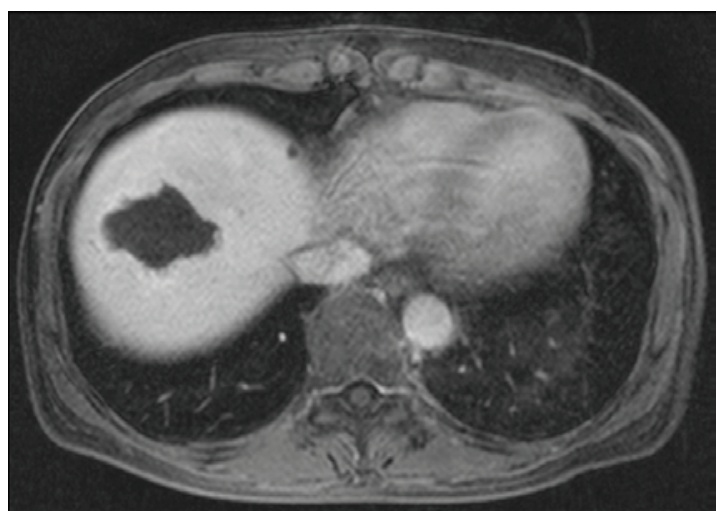

G

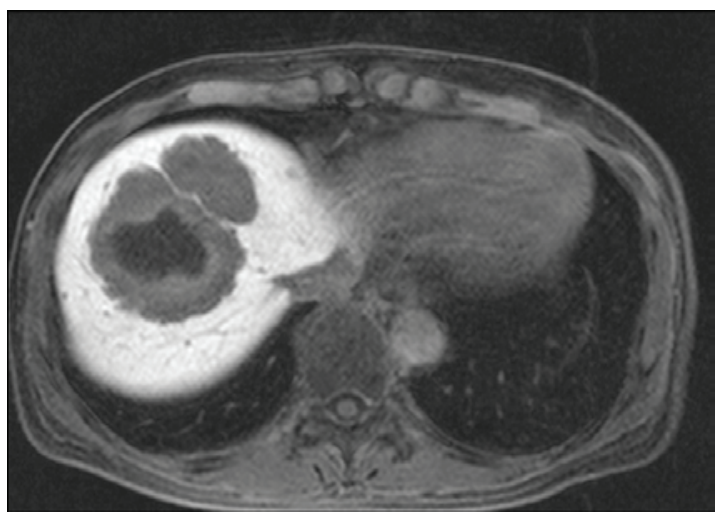

B

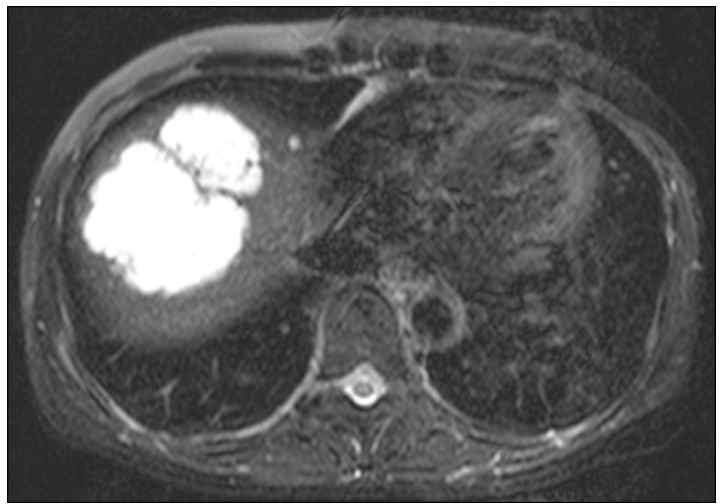

D

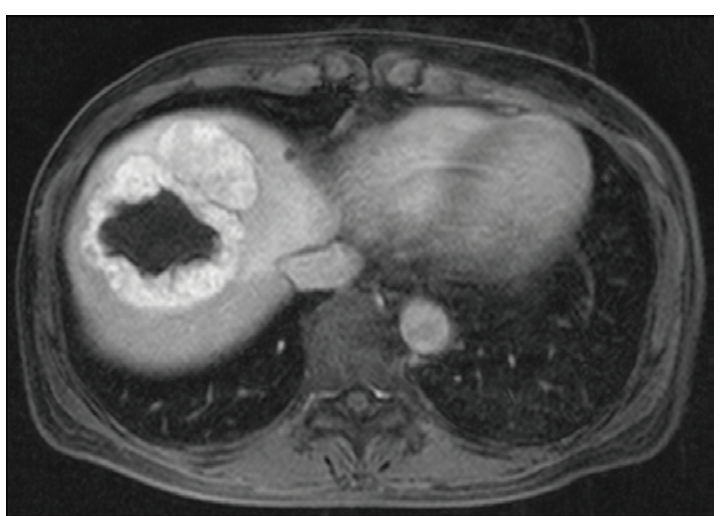

F

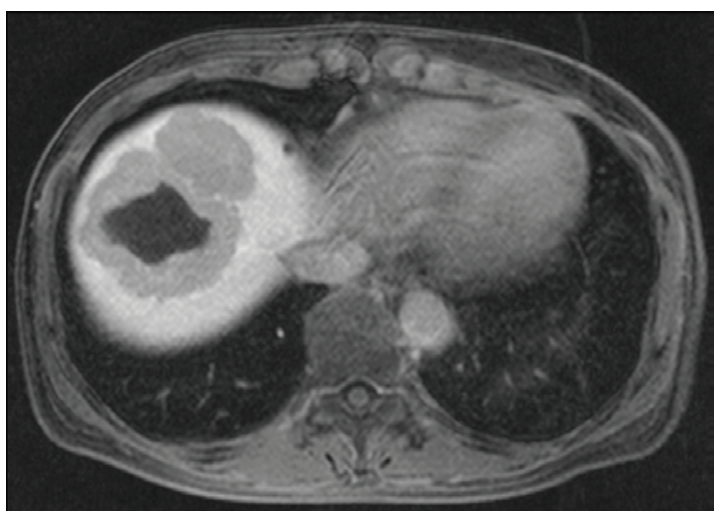

H

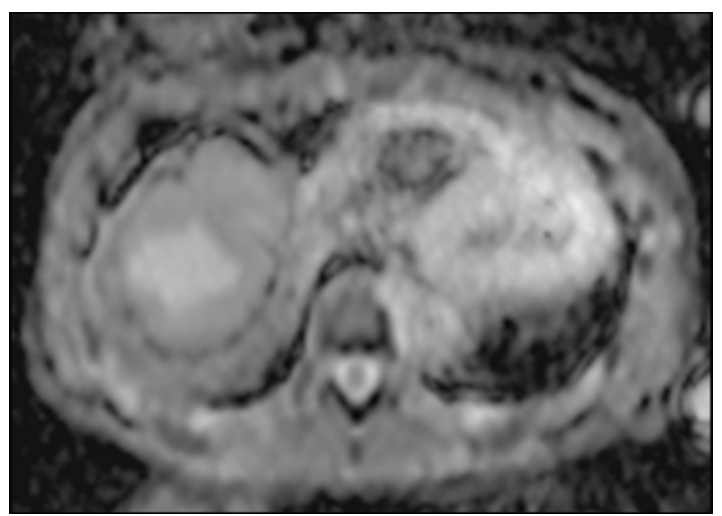

Figure 2. Magnetic resonance imaging (MRI) findings. (A) Tumor appears as a hypointense mass on T1-weighted image and (B) as hyperintensity on fatsuppression T2-weighted image, (C) prior to enhancement. Following the administration of Gd-EOB-DTPA, (D) the tumor shows marked enhancement in the arterial phase, (E) iso-isointensity in the portal phase, and (F) hypointensity in the equilibrium phase. (G) The hepatobiliary phase reveals hypointensity, and there is no enhanced area inside the tumor. (H) The mean apparent diffusion coefficient (ADC) value was $2.4 \times 10^{-3} \mathrm{~mm}^{2} / \mathrm{sec}$.

that the mean ADC value of benign tumors, including hemangiomas, $\left(2.45 \times 10^{-3} \pm 0.96 \mathrm{~mm}^{2} / \mathrm{sec}\right)$ was significantly higher than that of malignant lesions $\left(1.08 \times 10^{-3} \pm 0.50 \mathrm{~mm}^{2} / \mathrm{sec}\right)(14)$.
In the present case, the ADC values of IH ranged from 2.0 to $2.4 \times 10^{-3} \mathrm{~mm}^{2} / \mathrm{sec}$ and were similar to those of previous studies on hemangiomas. 


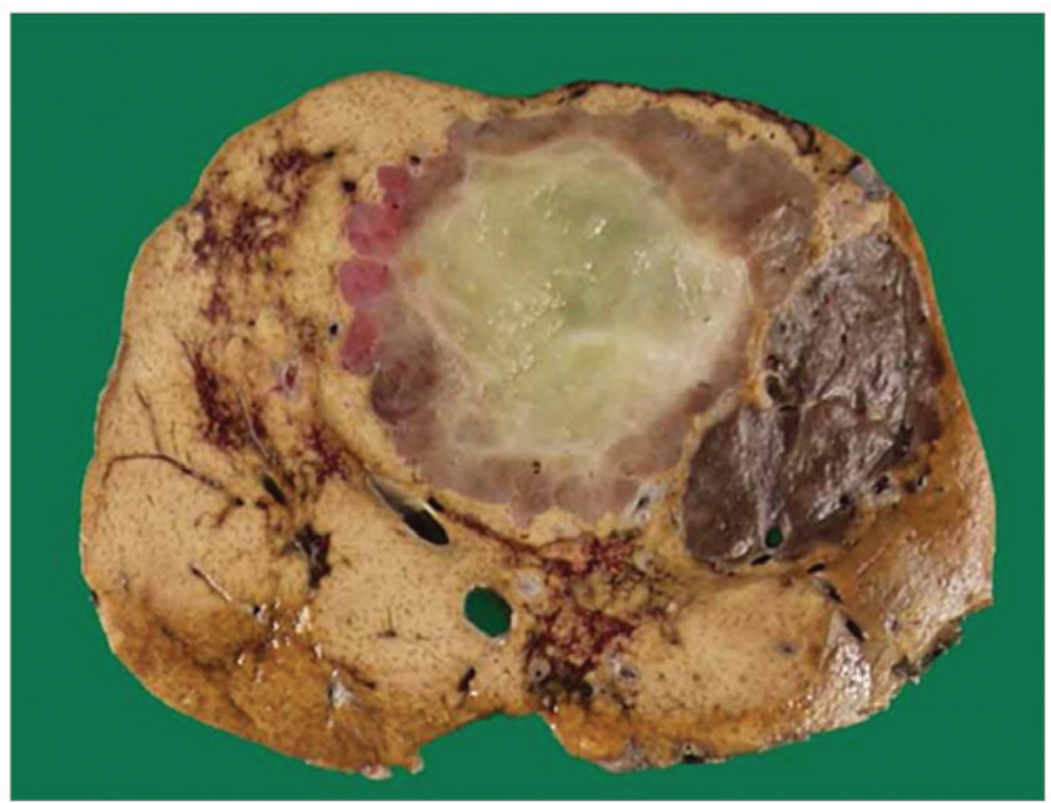

Figure 3. Macroscopic findings. The tumor is well defined and brown to tan, with a low color area considered to be myxoid degeneration.
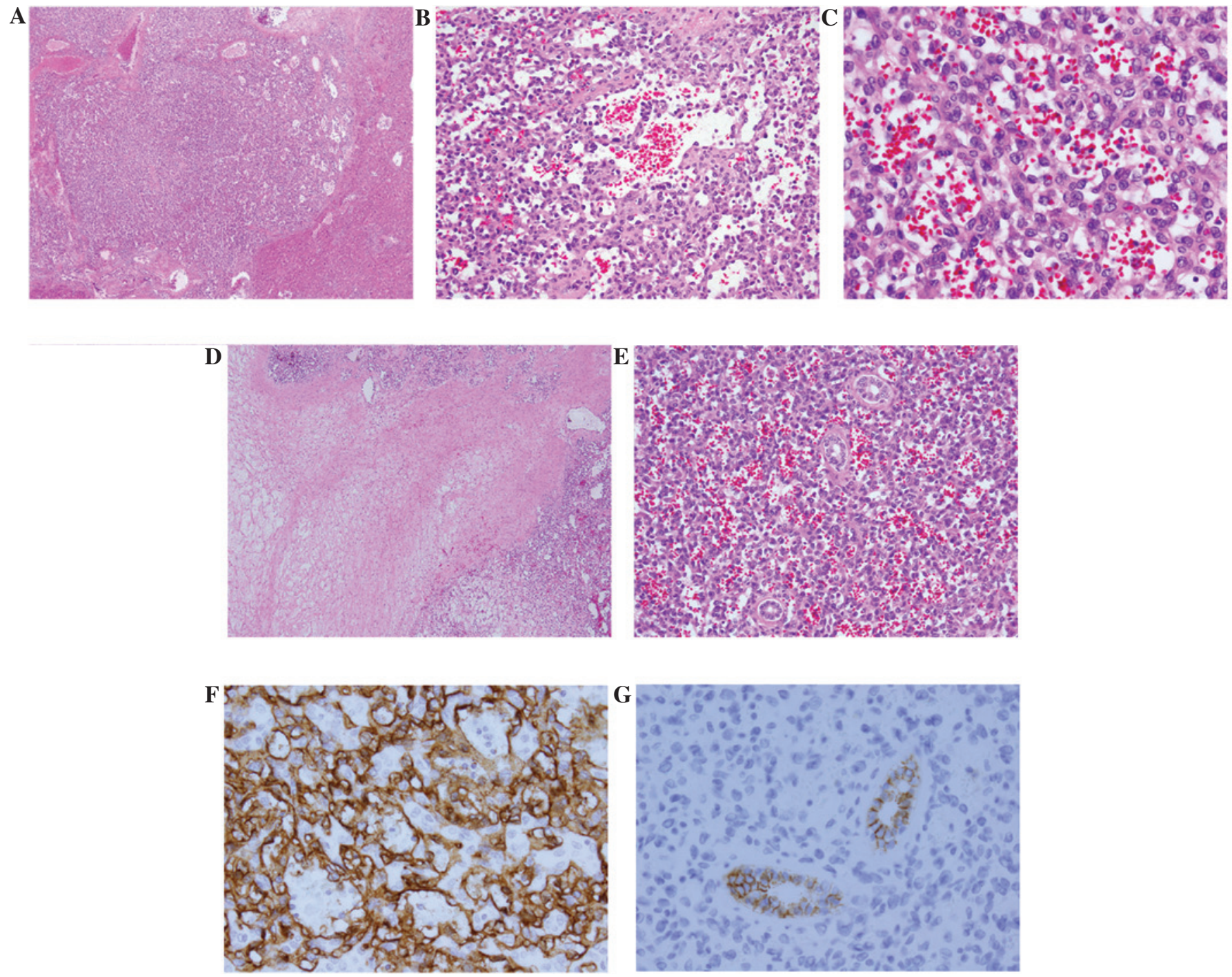

Figure 4. Microscopic findings. (A-C) The tumor consists of numerous capillary-like vessels lined by endothelial cells without atypia or mitoses. (D) Myxomatous component occurs in the center of one of the tumors. (F) Immunohistochemically, the endothelium was covered with vessels positive for CD34. (E and G) Small bile ducts, positive for CD56, trapped within the tumor are visible. 
Pathological studies on IH revealed that the tumor margin is macroscopically well demarcated in $65 \%$ of IH cases, and the cut surface is red-brown to light tan or white depending on the degree of vascularity, hemorrhaging or degeneration $(1,5,15)$. Multiple occurrence is observed in $45 \%$ of IH cases $(1,5)$. Those findings are also consistent with this case. Microscopically, IH tumors are composed of numerous capillary-like small vessels lined by plump endothelial cells, usually arranged in a single layer without mitoses $(3,4,15-18)$. The periphery of these lesions may also exhibit a certain degree of cavernous changes (18). Exclusively in IHs, small bile ducts are trapped and scattered within the tumor. Extramedullary hematopoiesis is also often observed $(4,15,17,18)$. Involutional changes, such as infarction, necrosis, hemorrhaging, scarring, myxoid changes and calcifications are frequently present $(4,17,18)$. Immunohistochemically, the tumor cells express CD31, CD34 and factor VIII-related antigen $(1,4,5,15,18)$. The majority of these findings were observed in the present case.

Angiosarcomas, composed of a vascular channel and typically occurring in adults, should be considered as a different diagnosis. However, angiosarcomas are usually composed of an irregular vascular channel lined by variably atypical endothelial cells and often exhibit multilayering and high mitotic activity (4). There were no atypical cells or mitoses in the present case. Angiosarcoma is usually a highly aggressive tumor and its prognosis is extremely poor. The present case had a clinically favorable outcome. By contrast, small bile ducts were trapped within the tumor. This finding is rare in a cavernous hemangioma, which is a representative benign vascular tumor occurring in adults. Collectively, these findings suggest that this case may be $\mathrm{IH}$ occurring in adults. IH-like tumors occur in various organs, however, they usually demonstrate a positive reaction immunohistochemically for Glut-1 (19). The present case lacked a positive reaction for Glut-1 in spite of exhibiting typical morphological findings of IH. Although these discrepancies remain unclear, the lack of Glut-1 expression may be a characteristic immunophenotype in adult onset IH. However, little is known about the Glut-1 status in adult onset IH owing to its rarity.

There are therapeutic methods available. The first-line therapy involves drugs such as steroids or interferon- $\alpha$. Arterial embolization, surgical resection or liver transplantation are selected if medication is ineffective $(4,6,7)$.

In conclusion, we presented a case of $\mathrm{IH}$ of the liver in an adult. $\mathrm{IH}$ is one of the most common benign liver tumors in infants and children. Although IH is a biologically benign tumor, its mortality rate is relatively high in patients with complications. Thus, radiologists and pathologists should understand the characteristics of IH in order to administer appropriate treatment.

\section{References}

1. Selby DM, Stocker JT, Waclawiw MA, Hitchcock CL and Ishak KG: Infantile hemangioendothelioma of the liver. Hepatology 20: 39-45, 1994.

2. Woltering MC, Robben S and Egeler RM: Hepatic hemangioendothelioma of infancy: treatment with interferon alpha. J Pediatr Gastroenterol Nutr 24: 348-351, 1997.

3. Diment J, Yurim O and Pappo O: Infantile hemangioendothelioma of the liver in an adult. Arch Pathol Lab Med 125: 931-932, 2001.

4. Bosman FT, Carineiro F, Hruban RH and Theise ND (eds): WHO Classification of Tumors of the Digestive System. 4th edition. World Health Organisation, Geneva, pp242-243, 2010.

5. Burt AD, Portmann BC and Ferrell LD (eds): MacSween's Pathology of the liver. 6th edition. Elsevier Limited, Philadelphia, PA, pp796-797, 2012.

6. Kuntz E and Kuntz HD: Hepatology Textbook and Atlas Third edition. Springer Medizin Verlag, Heidelberg, pp781-782, 2008.

7. Kassarjian A, Zurakowski D, Dubois J, Paltiel HJ, Fishman SJ and Burrows PE: Infantile hepatic hemangiomas: clinical and imaging findings and their correlation with therapy. AJR Am J Roentgenol 182: 785-795, 2004.

8. Mortele KJ, Mergo PJ, Urrutia M and Ros PR: Dynamic gadolinium-enhanced MR findings in infantile hepatic hemangioendothelioma. J Comput Assist Tomogr 22: 714-717, 1998.

9. Keslar PJ, Buck JL and Selby DM: From the archives of the AFIP. Infantile hemangioendothelioma of the liver revisited. Radiographics 13: 657-670, 1993.

10. Feng ST, Chan T, Ching AS, Sun CH, Guo HY, Fan M, Meng QF and Li ZP: CT and MR imaging characteristics of infantile hepatic hemangioendothelioma. Eur J Radiol 76: e24-e29, 2010.

11. Mortelé KJ, Vanzieleghem B, Mortelé B, Benoit Y and Ros PR: Solitary hepatic infantile hemangioendothelioma: dynamic gadolinium-enhanced MR imaging findings. Eur Radiol 12: $862-865,2002$

12. Tamada T, Ito K, Yamamoto A, Sone T, Kanki A, Tanaka F and Higashi H: Hepatic hemangiomas: evaluation of enhancement patterns at dynamic MRI with gadoxetate disodium. AJR Am J Roentgenol 196: 824-830, 2011.

13. Vossen JA, Buijs M, Liapi E, Eng J, Bluemke DA and Kamel IR: Receiver operating characteristic analysis of diffusionweighted magnetic resonance imaging in differentiating hepatic hemangioma from other hypervascular liver lesions. J Comput Assist Tomogr 32: 750-756, 2008.

14. Taouli B, Vilgrain V, Dumont E, Daire JL, Fan B and Menu Y: Evaluation of liver diffusion isotropy and characterization of focal hepatic lesions with two single-shot echo-planar MR imaging sequences: prospective study in 66 patients. Radiology 226: 71-78, 2003.

15. Ishak KG, Goodman ZD and Stocker TJ: Atlas of Tumor Pathology. Tumors of the Liver and Intrahepatic Bile Ducts. Armed Forces Institute of Pathology, Washington, D.C., 2001.

16. Dachman AH, Lichtenstein JE, Friedman AC and Hartman DS: Infantile hemangioendothelioma of the liver: a radiologicpathologic-clinical correlation. AJR Am J Roentgenol 140: 1091-1096, 1983

17. Okuda K and Ishak KG: Neoplasms of the Liver. Springer-Verlag, Tokyo, 1987.

18. Kanel GC and Korula J: Atlas of Liver Pathology. 3rd edition. Elsevier Saunders, Philadelphia, pp260-261, 2011.

19. Drut RM and Drut R: Extracutaneous infantile haemangioma is also Glut1 positive. J Clin Pathol 57: 1197-1200, 2004. 